\title{
Synthesis of Novel Series of Various Substituted1-(5-(2-p-tolyloxyquinolin-3-yl)-2- (pyridin-4-yl)-1,3,4-oxadiazol-3(2H)-yl)ethanone and its Antibacterial Activity
}

\author{
Ratnadeep S. Joshi, Priyanka G. Mandhane, Asha V. Chate, Charansingh H. Gill* \\ Department of Chemistry, Dr. Babasaheb Ambedkar Marathwada University, Aurangabad 431004, India \\ ${ }^{*}$ E-mail: rtndpjoshi@gmail.com
}

(Received February 1, 2011; Accepted July 8, 2011)

\begin{abstract}
A new series of 1-(5-(2-tolyloxyquinoline-3-yl)-2-(pyridine-4-yl)-1,3,4-oxidiazol-3(2H)-yl)ethanones were synthesized from cyclisation of $N^{\prime}$-((2-( $p$-tolyloxy)quinoline-3-yl)methylene) isonitonohydrazide in acetic unhydride at reflux condition for 3-4 hr. The structures of the new compounds were confirmed by elemental analyses as well as IR, ${ }^{1} \mathrm{H}$ NMR and mass spectral data. All the synthesized compounds were screened for their antibacterial activities against various bacterial strains. Several of these compounds showed potential antibacterial activity.
\end{abstract}

Key words: 1,3,4 oxadiazole, Quinoline, Isoniazide, Cyclisation, Antibacterial activity

\section{INTRODUCTION}

Quinoline ring systems represent a major class of heterocycles as they occur in various natural products especially in alkaloids. ${ }^{1}$ It possesses diverse biological and physiological activities such as antimalarial, ${ }^{2}$ anti-inflammatory, ${ }^{3}$ antitumor, ${ }^{4}$ DNA binding capacity, ${ }^{5}$ antibacterial properties. ${ }^{6}$ Recently, quinoline has been employed in the study of bio-organic and bio-organometallic processes. ${ }^{7}$ There are many quinoline-based compounds which are known to exhibit anti-TB properties. Compound TMC $207^{8}$ bearing a bulky biaryl side chain at position $\mathrm{C} 3$, is a highly potent anti-TB agent and is currently in phase II clinical trials. In particular 2-chloroquinoline-3-carbaldehyde has been used as a key intermediate for the synthesis of variety of medicinally valuable compounds. ${ }^{9}$

1,3,4-oxadiazole is widely exploited for various applications. A number of therapeutic agents such as HIV-integrase inhibitor raltegravir, a nitrofuran antibacterial furamizole, a potent PDF inhibitor BB-83698, antihypertensive agents Tiodazosin and Nesapidil are based on 1,3,4-oxadiazole moiety. The 1,3,4-oxadiazole undergoes number of reactions including elctrophillic substitution, nucleophilic substitution, thermal and photochemical. The substituted oxadiazoles are heterocyclic compounds, which serve both as biomimetic and reactive pharamacophores and many are key elements with potential biological activities ${ }^{10-12}$ such as pesticidal, ${ }^{13}$ antiperipheral vasomotility, ${ }^{14} \mathrm{CNS}$ stimulant, anti-inflammatory, hypertensive, ${ }^{15}$ insecticidal, ${ }^{16}$ bactericidal,${ }^{17}$ hypoglycemic,${ }^{18-19}$ analgesic, anticonvulsive, antiemetic, diuretic, ${ }^{20}$ muscle relaxant, ${ }^{21-22}$ herbicidal ${ }^{23-24}$ and fungicidal activity. ${ }^{25-26}$

Isoniazide (INH) is widely applied as first-line drugs for the treatment of tuberculosis, usually in combination with other drugs. Modifying either of these molecules has been a challenge taken up by several research groups. ${ }^{27} \mathrm{INH}$ prevents a mycolic acid biosynthesis by inhibiting a 2trans-enoyl-acyl carrier protein reductase (InhA) that belongs to the FAS-II (fatty acid synthetase II) system. ${ }^{28}$

In our continuous program in the search of new potent and safe synthesis of biologically active heterocycles, ${ }^{29-30}$ we have planned to synthesize some new series of various substituted 1-(5-(2-( $p$-tolyloxy)quinoline-3-yl)-2-(pyridine4-yl)-1,3,4-oxadiazol-3(2H)-yl)ethanones by oxidative cyclisation of $\mathrm{N}$-((2-( $p$-tolyloxy)quinoline-3-yl)methylene)isonitonohydrazide using by cyclisation using acetic anhydride under reflux condition and evaluating their antibacterial activity against the standard drug Streptomycin and Ampicillin.

\section{RESULT AND DISCUSSION}

\section{Chemistry}

The synthetic work was carried out beginning from 2chloro-3-formylquinoline $\mathbf{1}(\mathbf{a}-\mathbf{j})$ according to Scheme 1 . The reaction of 2-chloro-3-formylquinoline 1(a-j) with $p$ Cresol in presence of $\mathrm{K}_{2} \mathrm{CO}_{3} / \mathrm{DMF}$ at $80-90{ }^{\circ} \mathrm{C}$ for $4-5 \mathrm{hr}$ gave substituted 2-( $p$-tolyoxy)quinoline-3-carbaldehyde $\mathbf{2}(\mathbf{a}-\mathbf{j})$. Further reaction of isonicotinohydrazide 3 with substituted 2-( $p$-tolyoxy)quinoline-3-carbaldehyde 2(a-j) 

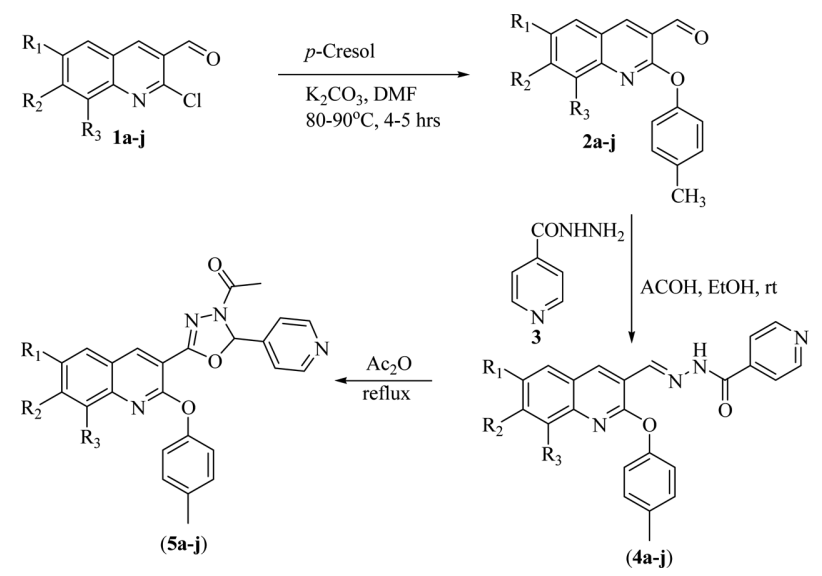

Scheme 1.

gave corresponding $N^{\prime}-((2-(p$-tolyloxy)quinoline-3-yl) methylene)isonitonohydrazide $\mathbf{4}(\mathbf{a}-\mathbf{j})$ in acetic acid in ethanol at room temperature. followed by cyclisation of $N$ ((2-( $p$-tolyloxy)quinoline-3-yl)methylene)isonitonohydrazide using acetic anhydride to gave our targeted product i.e. 1-(5-(2-( $p$-tolyloxy)quinoline-3-yl)-2-(pyridine-4yl)-1,3,4-oxadiazol-3(2H)-yl)ethanone 5(a-j). All the synthesized compounds were characterized by their physical and spectral data (IR, ${ }^{1} \mathrm{H}-\mathrm{NMR}$, Mass).

\section{Spectral analysis}

The structures of the synthesized compounds were confirmed by spectral analysis (IR, ${ }^{1} \mathrm{H}$ NMR and Mass). The IR spectrum of compound 2a showed a peak at $1690 \mathrm{~cm}^{-1}$ due to $\mathrm{C}=\mathrm{O}$ stretch. In ${ }^{1} \mathrm{H}$ NMR spectrum it exhibited two singlets, one at $\delta 2.41$ due to $-\mathrm{CH}_{3}$ protons and second at $\delta 10.65$ due to $-\mathrm{CHO}$ proton. Mass spectrum was consistent with assigned structure showing $\mathrm{M}+1$ ) peak at $\mathrm{m} / \mathrm{z}=$ 264.1. The structure of $\mathbf{4 a}$ is interpreted from spectroscopic data, IR spectra of compound $\mathbf{4 a}$ reveals absorption band in the region $1560 \mathrm{~cm}^{-1}$ corresponding to $-\mathrm{C}=\mathrm{N}$ stretching at $1640 \mathrm{~cm}^{-1}$. The ${ }^{1} \mathrm{H}$ NMR spectra of $4 \mathbf{a}$ exhibits a sharp singlet at $\delta 2.01$ for $-\mathrm{CH}_{3}$ and one broad singlet at $\delta 10.50$ of $-\mathrm{NH}$. The pyridine ring protons appeared doublet at $\delta 8.92$ and 8.75 respectively. $\mathrm{C}_{4}$ - proton of quinoline nuclei appears as a singlet at $\delta 8.33$. All other aromatic protons arise at respective position. The IR spectrum of compound 5a showed absorption peak at $1510 \mathrm{~cm}^{-1}$ due to $\mathrm{C}=\mathrm{N}$ stretching vibrations. The peak at $1665 \mathrm{~cm}^{-1}$ due to the $\mathrm{N}-\mathrm{COCH}_{3}$ confirms the formation of $\mathrm{N}$-acyl oxadiazole ring, its ${ }^{1} \mathrm{H}$ NMR spectrum revealed a singlet at $\delta 4.61$ due to $\mathrm{CO}-\mathrm{CH}_{3}$ also one singlet appears at 6.95 ppm due - $\mathrm{CH}$ of oxadiazole ring and rest of the protons appears at repective position.
Table 1. Physical data of the compounds 5(a-j)

\begin{tabular}{cccccc}
\hline Comp. No. & $\mathrm{R}_{1}$ & $\mathrm{R}_{2}$ & $\mathrm{R}_{3}$ & Yield $(\%)$ & M.P. $\left({ }^{\circ} \mathrm{C}\right)$ \\
\hline $\mathbf{5 a}$ & $\mathrm{H}$ & $\mathrm{H}$ & $\mathrm{H}$ & 51 & Sticky \\
$\mathbf{5 b}$ & $\mathrm{CH}_{3}$ & $\mathrm{H}$ & $\mathrm{H}$ & 56 & $185-186$ \\
$\mathbf{5 c}$ & $\mathrm{H}$ & $\mathrm{CH}_{3}$ & $\mathrm{H}$ & 46 & Sticky \\
$\mathbf{5 d}$ & $\mathrm{H}$ & $\mathrm{H}$ & $\mathrm{CH}_{3}$ & 42 & $161-162$ \\
$\mathbf{5 e}$ & $\mathrm{OCH}_{3}$ & $\mathrm{H}$ & $\mathrm{H}$ & 54 & $144-145$ \\
$\mathbf{5 f}$ & $\mathrm{H}$ & $\mathrm{OCH}_{3}$ & $\mathrm{H}$ & 57 & $191-192$ \\
$\mathbf{5 g}$ & $\mathrm{H}$ & $\mathrm{H}$ & $\mathrm{OCH}_{3}$ & 54 & $163-164$ \\
$\mathbf{5 h}$ & $\mathrm{OC}_{2} \mathrm{H}_{5}$ & $\mathrm{H}$ & $\mathrm{H}$ & $60-$ & $157-158$ \\
$\mathbf{5 i}$ & $\mathrm{H}$ & $\mathrm{OC}_{2} \mathrm{H}_{5} \mathrm{H}$ & $\mathrm{H}$ & 61 & $178-179$ \\
$\mathbf{5 j}$ & $\mathrm{H}$ & $\mathrm{H}$ & $\mathrm{OC}_{2} \mathrm{H}_{5}$ & 47 & $156-157$ \\
\hline \multicolumn{5}{r}{}
\end{tabular}

\section{Antibacterial activity}

The antibacterial activities of the synthesized compounds were determined by the well-diffusion method. ${ }^{31}$ In present work, two Gram positive bacteria, Bacillus subtilis (ATCC 6633), Staphylococcus aureus (ATCC 25923) and two Gram negative bacteria, Salmonella typhimurium (ATCC 23564), Pseudomonas aeruginosa (ATCC 27853) were used to investigate the antibacterial activities. The bacterial liquid cultures were prepared in fusion broth for their activity tests. The compounds were dissolved in DMSO at concentration of $1 \mathrm{mgmL}^{-1}$. Antibacterial activity of DMSO against the test organisms was investigated, and was found to be nil. Molten nutrient agar $\left(15 \mathrm{~cm}^{3}\right)$ kept at $45^{\circ} \mathrm{C}$, was then poured into the Petri dishes and allowed to solidify. Ten millimeter diameter holes were then punched carefully using a sterile cork borer and completely filled with the test solutions. The plates were incubated for $24 \mathrm{hr}$ at $37^{\circ} \mathrm{C}$. After $24 \mathrm{hr}$, the inhibition zone that appeared around the holes in each plate was measured. Antibacterial activity was determined by measuring the diameter of inhibition zone and examining the minimal inhibitory concentration (MIC). Activity of each compound was compared with streptomycin and ampicillin as standards. The observed data of antibacterial activity of compounds and the standard drugs is given in Table 2. The compounds $\mathbf{5 e}, \mathbf{5 f}, \mathbf{5 i}$ and $\mathbf{5 j}$ show excellent antibacterial activity against Gram positive bacterial strains. Likewise compounds $\mathbf{5 a}, \mathbf{5 c}$ and 5g showed excellent activity against Gram negative bacterial strains.

\section{EXPERIMENTAL SECTION}

Melting points were recorded in open capillaries in liquid paraffin bath and are uncorrected. The progress of reaction was monitored by thin layer chromatography using silica gel (Merck). IR spectra were recorded on a SHI- 
Table 2. The Minimum Inhibitor concentrations of tested compounds 5(a-j) in $\mu \mathrm{g} / \mathrm{mL}$

\begin{tabular}{|c|c|c|c|c|}
\hline Tested Compounds & B. subtilis $\mathrm{ZI}^{a}(\mathrm{MIC})^{b}$ & S. aureus $\mathrm{ZI}^{a}(\mathrm{MIC})^{b}$ & S. typhi ZI ${ }^{a}(\mathrm{MIC})^{b}$ & P. aeroginosa $\mathrm{ZI}^{a}(\mathrm{MIC})^{b}$ \\
\hline $5 \mathrm{a}$ & $13.4(15)$ & $13.7(15)$ & $15.1(10)$ & $14.8(10)$ \\
\hline $5 b$ & 13.1(15) & $13.2(15)$ & $11.1(20)$ & $10.4(20)$ \\
\hline $5 c$ & $13.8(15)$ & $13.3(15)$ & $15.1(10)$ & $14.9(10)$ \\
\hline $5 d$ & $14.3(10)$ & 14.1(10) & $12.8(15)$ & $12.7(15)$ \\
\hline $5 e$ & $14.8(10)$ & $14.6(10)$ & $12.9(15)$ & $12.8(15)$ \\
\hline $5 f$ & $15.1(10)$ & $14.7(10)$ & $13.5(15)$ & $13.4(15)$ \\
\hline $5 \mathrm{~g}$ & $13.2(15)$ & $13.5(15)$ & $14.8(10)$ & $14.5(10)$ \\
\hline $5 \mathrm{~h}$ & $14.1(10)$ & $14.6(10)$ & $12.2(15)$ & $12.6(15)$ \\
\hline $5 \mathrm{i}$ & $15.6(10)$ & $15.3(10)$ & $11.7(15)$ & $11.2(15)$ \\
\hline $5 \mathrm{j}$ & $15.1(10)$ & $15.4(10)$ & $11.2(15)$ & $11.3(15)$ \\
\hline Streptomycin & $15.1(10)$ & $14.9(10)$ & $16.4(5)$ & $16.1(5)$ \\
\hline Ampicillin & $14.3(10)$ & $14.7(10)$ & $16.3(5)$ & $15.9(5)$ \\
\hline
\end{tabular}

${ }^{a}$ Zone of inhibition, ${ }^{b}$ Minimum inhibitory concentration in $\mu \mathrm{g} / \mathrm{mL}$

MADZU-FT-IR spectrophotometer in $\mathrm{KBr}$ disc. ${ }^{1} \mathrm{H}$ NMR spectra were recorded on BRUKER ADVANCE-II 400 NMR spectrophotometer in DMSO- $d_{6}$ as a solvent and TMS as an internal standard. Peak values are shown in $\mathrm{d}$ ppm. Mass spectra were recorded on a PEP-SCIUX-APIQ pulsar (electron pre-ionization) mass spectrometer. Elemental analyses were performed on Perkin-Elmer EAL240 elemental analyzers.

General procedure for the synthesis of 2 -(p-tolyloxy)quinoline-3-carbaldehydes $\mathbf{2}(\mathbf{a}-\mathbf{j})$

To the mixture of $p$-cresol (3.7 gm, $0.034 \mathrm{~mol}$ ) and $\mathrm{K}_{2} \mathrm{CO}_{3}$ (12.8 gm, $0.093 \mathrm{~mol}$ ) in $\mathrm{DMF}$, compound 1a (6 gm, $0.031 \mathrm{~mol}$ ) was added and the reaction mixture was stirred at $80-90{ }^{\circ} \mathrm{C}$ for $4-5 \mathrm{hr}$. The completion of the reaction was monitored on TLC. After completion of the reaction, ice cold water $(50 \mathrm{ml})$ was poured on the reaction mixture \& the solid thus obtained was filtered off and washed with water, further the compound was recrystallized in ethyl acetate.

\section{2-(p-tolyloxy)quinoline-3-carbaldehyde (2a)}

IR-(KBr, cm $\left.{ }^{-1}\right): 1690(\mathrm{C}=\mathrm{O}), 1610,1525(\mathrm{C}=\mathrm{C})$; ${ }^{1} \mathrm{H}$ NMR$\left(400 \mathrm{~Hz}, \mathrm{DMSO}-d_{6}\right): \delta 10.65(\mathrm{~s}, 1 \mathrm{H},-\mathrm{CHO}), 8.72(\mathrm{~s}, 1 \mathrm{H}$, Ar-H), 7.89(d, $J=8 \mathrm{~Hz}, 1 \mathrm{H}, \mathrm{Ar}-\mathrm{H}), 7.73(\mathrm{~d}, J=8 \mathrm{~Hz}, 1 \mathrm{H}$, Ar-H), 7.70(t, 1H, Ar-H), 7.45(t, 1H, Ar-H), 7.27(d, $J=8$ $\mathrm{Hz}, 2 \mathrm{H}, \mathrm{Ar}-\mathrm{H}), 7.18(\mathrm{~d}, J=8 \mathrm{~Hz}, 2 \mathrm{H}, \mathrm{Ar}-\mathrm{H}), 2.41(\mathrm{~s}, 3 \mathrm{H}$, $\left.-\mathrm{CH}_{3}\right)$; ES-MS (m/z): $264.4(\mathrm{M}+1)$.

General procedure for the synthesis of (E)-N'-((2-tolyloxy) quinoline-3-yl) methylene) isonicotinohydrazide 4(a-j)

An equimolar mixture of 2-chloro-3-formyl quinoline
$1 \mathbf{a}(0.004 \mathrm{~mol})$ and isonicotinohydrazide $3(0.004 \mathrm{~mol})$ in $10 \mathrm{~mL}$ ethanol containing few drops of glacial acetic acid was strired at room temperature. After completion of reaction (checked by TLC), the excess of solvent was removed on rotary evaporator to yield solid which was washed with petroleum ether followed by crystallization from ethanol.

(E)-N'-((2-tolyloxy) quinoline-3-yl) methylene)isonicotinohydrazide (4a)

IR (KBr, cm $\left.{ }^{-1}\right): 2980(-\mathrm{NH}), 1560(\mathrm{C}=\mathrm{N}), 1513(\mathrm{C}=\mathrm{C})$; ${ }^{1} \mathrm{H}$ NMR (400 Hz, DMSO- $\left.d_{6}\right): \delta 10.72$ (s, $\left.1 \mathrm{H},-\mathrm{NH}\right), 8.90$ (s, 1H), 8.77 (d, $J=7.92 \mathrm{~Hz}, 3 \mathrm{H}, \mathrm{Ar}-\mathrm{H}), 7.75-7.80$ (m, 4H, Ar-H), 7.26 (t, $J=10.94 \mathrm{~Hz}, 2 \mathrm{H}, \mathrm{Ar}-\mathrm{H}), 7.19$ (dd, $J=8.21$ \& $4.24 \mathrm{~Hz}, 4 \mathrm{H}, \mathrm{Ar}-\mathrm{H}) 2.30$ (s, 3H, Ar- $\left.\mathrm{CH}_{3}\right), \mathrm{ES}-\mathrm{MS}$ (m/z): 383(M+1); Anal. Calcd. $\mathrm{C}_{23} \mathrm{H}_{18} \mathrm{~N}_{4} \mathrm{O}_{2} \mathrm{C}, 72.24 ; \mathrm{H}, 4.74 ; \mathrm{N}$, 14.65, found $\mathrm{C}, 72.22 ; \mathrm{H}, 4.80 ; \mathrm{N}, 14.56 \%$.

(E)-N'-((2-tolyloxy)-6-ethoxyquinoline-3-yl)methylene)isonicotinohydrazide (4h)

IR $\left(\mathrm{KBr}, \mathrm{cm}^{-1}\right): 3009(-\mathrm{NH}), 1577(\mathrm{C}=\mathrm{N}), 1510(\mathrm{C}=\mathrm{C})$; ${ }^{1} \mathrm{H}$ NMR (400 Hz, DMSO-d $)$ ): $\delta 10.79$ (s, 1H, -NH), 8.92 (s, 1H), 8.73 (d, $J=8.21 \mathrm{~Hz}, 2 \mathrm{H}, \mathrm{Ar}-\mathrm{H}) 7.84-7.89$ (dd, $4 \mathrm{H}$, Ar-H), 7.21 (t, $J=11.30 \mathrm{~Hz}, 2 \mathrm{H}, \mathrm{Ar}-\mathrm{H}), 7.16$ (dd, $J=8.44$ \& 4.66Hz, 4H, Ar-H), 2.67 (s, 3H, Ar- $\left.\mathrm{CH}_{3}\right), 1.41$ (s, 3H, $\left.-\mathrm{CH}_{3}\right)$; ES-MS (m/z): $427(\mathrm{M}+1)$; Anal. Calcd. $\mathrm{C}_{25} \mathrm{H}_{22} \mathrm{~N}_{4} \mathrm{O}_{3}$ C, 70.41; H, 5.20; N, 13.14, found C, 70.43; H, 5.30; N, $13.21 \%$.

General Procedure for the Synthesis of Substituted 1(5-(2-(p-tolyloxy)quinoline-3-yl)-2-(pyridine-4-yl)-1,3,4oxadiazol-3(2H)-yl)ethanones $5(\mathbf{a}-\mathbf{j})$

$N^{\prime}$-((2-(p-tolyloxy)quinoline-3-yl)methylene)isonicitinohydrazide $(0.1 \mathrm{gm}, 0.00028 \mathrm{~mol})$ 4a was dissolved in 
$10 \mathrm{ml}$ of acetic anhydride. The reaction mixture was heated in an oil bath for 3-4 h at $120^{\circ} \mathrm{C}$ and left for overnight. $10 \mathrm{ml}$ cold water was slowly added to the flask and the separated product was filtered and washed with water, dried under vacuum and recrystallized from ethanol.

1-(5-(2-(p-tolyloxy)quinolin-3-yl)-2-(pyridin-4-yl)-1,3,4oxadiazol-3(2H)-yl)ethanone (5a)

IR KBr: $1665\left(\mathrm{~N}-\mathrm{CO}-\mathrm{CH}_{3}\right) ; 1610(\mathrm{C}=\mathrm{N}) \mathrm{cm}^{-1} ;{ }^{1} \mathrm{H} \mathrm{NMR}$ (400 MHz, $\left.\mathrm{CDCl}_{3}\right): \delta 2.332$ (s, 3H, $\left.\mathrm{Ar}_{-} \mathrm{CH}_{3}\right) ; 4.621$ (s, 3H, $\left.\mathrm{N}-\mathrm{CO}-\mathrm{CH}_{3}\right) ; 6.956(\mathrm{~s} ; 1 \mathrm{H},-\mathrm{CH}) ; 7.115$ (d, J=8.0 Hz, 2H, Ar-H); 7.244 (d, J=8.0 Hz, 2H, Ar-H); 7.623-7.851 (m, $6 \mathrm{H}, \mathrm{Ar}-\mathrm{H}) ; 8.151$ (s, $1 \mathrm{H}$, quinoline $\left.\mathrm{C}_{4}-\mathrm{H}\right) ; 9.022$ (s, 2H, pyridine); $\operatorname{Mass}(\mathrm{m} / \mathrm{z}): 425(\mathrm{M}+1)$.

1-(5-(2-(p-tolyloxy)-6-methylquinolin-3-yl)-2-(pyridin-4-yl)-1,3,4-oxadiazol-3(2H)-yl) ethanone (5b)

IR KBr: $1688\left(\mathrm{~N}-\mathrm{CO}-\mathrm{CH}_{3}\right) ; 1593(\mathrm{C}=\mathrm{N}) \mathrm{cm}^{-1} ;{ }^{1} \mathrm{H} \mathrm{NMR}$ (400 MHz, DMSO): $\delta 2.344\left(\mathrm{~s}, 3 \mathrm{H}, \mathrm{Ar}-\mathrm{CH}_{3}\right) ; 2.275$ (s, $\left.3 \mathrm{H}, \mathrm{Ar}-\mathrm{CH}_{3}\right) ; 3.829$ (s, 3H, $\left.\mathrm{NCOCH}_{3}\right) ; 6.945$ (s, 1H, -CH); 7.107-7.166 (m, 3H, Ar-H); 7.275 (d, J=8.4 Hz, 2H, Ar-H); 7.861 (dd, $J=4.6 \& 6.0 \mathrm{~Hz}, 3 \mathrm{H}, \mathrm{Ar}-\mathrm{H}) ; 8.026$ (d, $J=8.8 \mathrm{~Hz}$, $1 \mathrm{H}$, Ar-H); 8.772-8.821(d, 2H, pyridine); 8.962(s, $1 \mathrm{H}$, quinoline $\left.\mathrm{C}_{4}-\mathrm{H}\right)$; $\operatorname{Mass}(\mathrm{m} / \mathrm{z})$ : 439.0(M+1).

1-(5-(2-(p-tolyloxy)-7-methylquinolin-3-yl)-2-(pyridin-4-yl)-1,3,4-oxadiazol-3(2H)-yl)ethanone (5c)

IR KBr: $1649\left(\mathrm{~N}-\mathrm{CO}-\mathrm{CH}_{3}\right) ; 1556(\mathrm{C}=\mathrm{N}) \mathrm{cm}^{-1} ;{ }^{1} \mathrm{H} \mathrm{NMR}$ (400 MHz, DMSO): $\delta 2.154$ (s, 3H, Ar-CH3); 2.165 (s, 3H, $\left.\mathrm{Ar}-\mathrm{CH}_{3}\right) ; 3.984\left(\mathrm{~s}, 3 \mathrm{H}, \mathrm{NCOCH}_{3}\right) ; 6.546(\mathrm{~s}, 1 \mathrm{H},-\mathrm{CH})$; 7.166-7.245 (m, 4H, Ar-H); 7.315 (d, $J=8.4$ Hz, 1H, ArH); 7.748 (dd, $J=3.4 \& 7.2 \mathrm{~Hz}, 3 \mathrm{H}, \mathrm{Ar}-\mathrm{H}) ; 8.126$ (d, $J=7.8$ $\mathrm{Hz}, 1 \mathrm{H}, \mathrm{Ar}-\mathrm{H})$; 8.845-8.875 (d, 2H, pyridine); 8.987 (s, $1 \mathrm{H}$, quinoline $\left.\mathrm{C}_{4}-\mathrm{H}\right)$; $\operatorname{Mass}(\mathrm{m} / \mathrm{z})$ : 439.3(M+1).

1-(5-(2-(p-tolyloxy)-7-methoxyquinolin-3-yl)-2-(pyridin-4-yl)-1,3,4-oxadiazol-3(2H)-yl)ethanone (5g)

IR KBr: $1687\left(\mathrm{~N}-\mathrm{CO}-\mathrm{CH}_{3}\right) ; 1510(\mathrm{C}=\mathrm{N}) \mathrm{cm}^{-1}$; ${ }^{1} \mathrm{H}$ NMR (400 MHz, DMSO): $\delta 2.54\left(\mathrm{~s}, 3 \mathrm{H}, \mathrm{Ar}-\mathrm{CH}_{3}\right) ; 3.651$ (s, 3H, $\left.\mathrm{Ar}-\mathrm{OCH}_{3}\right) ; 3.914$ (s, 3H, $\left.\mathrm{NCOCH}_{3}\right) ; 6.646$ (s, 1H, -CH); 6.924-7.145 (m, 3H, Ar-H); 7.245 (d, J=8.4 Hz, 2H, Ar$\mathrm{H}) ; 7.658(\mathrm{dd}, J=2.6 \& 6.8 \mathrm{~Hz}, 3 \mathrm{H}, \mathrm{Ar}-\mathrm{H}) ; 8.646$ (d, $J=8.8$ $\mathrm{Hz}, 1 \mathrm{H}, \mathrm{Ar}-\mathrm{H})$; 8.845-8.895 (d, 2H, pyridine); 8.912 (s, $1 \mathrm{H}$, quinoline $\left.\mathrm{C}_{4}-\mathrm{H}\right)$; $\operatorname{Mass}(\mathrm{m} / \mathrm{z})$ : 455.1(M+1).

1-(5-(2-(p-tolyloxy)-7-ethoxyquinolin-3-yl)-2-(pyridin-4-yl)-1,3,4-oxadiazol-3(2H)-yl)ethanone (5h)

IR KBr: $1667\left(\mathrm{~N}-\mathrm{CO}-\mathrm{CH}_{3}\right) ; 1556(\mathrm{C}=\mathrm{N}) \mathrm{cm}^{-1} ;{ }^{1} \mathrm{H} \mathrm{NMR}$ (400 MHz, DMSO): $\delta 2.464\left(\mathrm{~s}, 3 \mathrm{H}, \mathrm{Ar}-\mathrm{CH}_{3}\right) ; 2.563$ (t, $\left.3 \mathrm{H}, \mathrm{CH}_{2} \mathrm{CH}_{3}\right) ; 3.456$ (q, $\left.2 \mathrm{H}, \mathrm{OCH}_{2}\right) ; 3.814$ (s, 3H, $\mathrm{NCOCH}_{3}$ ); 6.512 (s, 1H, -CH); 6.958-7.165 (m, 3H, Ar-H); 7.145 (d, $J=7.2 \mathrm{~Hz}, 2 \mathrm{H}, \mathrm{Ar}-\mathrm{H}) ; 7.456$ (dd, $J=4.2 \& 7.6 \mathrm{~Hz}, 3 \mathrm{H}$, Ar$\mathrm{H}) ; 8.245$ (d, $J=8.2 \mathrm{~Hz}, 1 \mathrm{H}, \mathrm{Ar}-\mathrm{H}) ; 8.312$ (s, 1H, quinoline $\left.\mathrm{C}_{4}-\mathrm{H}\right) ; 8.614-8.814(\mathrm{~d}, 2 \mathrm{H}$, pyridine $) ; \operatorname{Mass}(\mathrm{m} / \mathrm{z})$ : $469.1(\mathrm{M}+1)$.

Acknowledgements. The authors are thankful to to The Head, Department of Chemistry, Dr. Babasaheb Ambedkar Marathwada University, Aurangabad, for his valuable support and laboratory facilities. And RSJ is thankful to University Grants Commission, New Delhi, for awarding the fellowship.

\section{REFERENCES}

1. (a) MethCohn, O.; Narine, B.; Tarnowski, B.; Hayes, R.; Keyzad, A.; Rhouti, S.; Robinson, A. J. Chem. Soc. Perkin Trans. 1981, 1, 2509 (b) Bhaduri, A. P. Synlett. 1990, 557.

2. (a) Mccormick, J. L.; Mckee, T. C.; Cardellina, J. H.; Boyd, M. R. J. Nat. Prod. 1996, 59, 469 (b) Chen, I. S.; Chen, H. F.; Cheng, M. J.; Chang, Y. L.; Teng, C. M.; Tsutomu, I.; Chen, J. J.; Tsai, I. L. J. Nat. Prod. 2001, 64, 1143 (c) Nadaraj, V.; Selvi, S. T.; Sasi, R. Arkivoc. 2006, $x, 82$.

3. Craig, J. C.; Person, P. E. J. Med. Chem. 1971, 14, 1221.

4. Dillard, R. D.; Pavey, D. E.; Benslay, D. N. J. Med Chem. 1973, 16, 251.

5. Sukhova, N. M.; Lidak, M.; Zidermane, A.; Pelevina, I. S.; Voronia, S. S. Khim. Farm. Zh. 1989, 23, 1226.

6. Atwell, G. J.; Bangaley, B. C.; Denny, W. A. J. Med. Chem. 1989, 32, 396.

7. Patel, H. V.; Vyas, K. V.; Fernandes, P. S. Ind. J. Chem. 1990, $29(B), 836$.

8. Saito, I.; Sando, S.; Nakatani, K. Bioorg. Med. Chem. 2001, 9, 2381.

9. Andries, K.; Verhasselt, P.; Guillemont, J.; Goehlmann, H. W. H.; Neefs, J. M.; Winkler, H.; Van Gestel, J.; Timmerman, P.; Zhu, M.; Lee, E.; Williams, P.; De Chaffoy, D.; Huitric, E.; Hoffner, S.; Cambau, E.; Truffot-Pernot, C.; Lounis, N.; Jarlier, V. A. Science, 2005, 307, 223.

10. Joseph, J. P.; Harry, L. Y. U.S.Patent 3,141,022, 1961; Chem. Abstr. 1964, 61, 8317b.

11. Hokfelt, B.; Jonsson, A. J. Med. Chem. 1962, 5, 247.

12. Ansel, P. S. U.S.Patent 2,883,391, 1959. Chem. Abstr. 1959, 53, 16157g.

13. Hiroshi, K.; Isaq, H.; Shigeki, O.; Zassokenkyn 1969, 8, 46; Chem. Abstr. 1970, 73, 108544 b.

14. Derappe, C.; Rips, R.; Albert, O. Aurousseau, M. Chim. Ther. 1968, 3, 181; Chem. Abstr. 1968, 69, 106626y.

15. Deshmukh, A. A.; Sattur, P. B.; Sheth, U. K. Indian. J. Exp. Biol. 1976, 4, 166. 
16. SenGupta, A. K.; Garg, M.; Chandra, U. J. Indian Chem. Soc. 1979, 56, 1230.

17. Chiyomaru, I.; Takita, K.; Ito, H.; Kumiai Chem. Ind. Co. Ltd. Jap. Pat. 1972, 72 07, 549; Chem. Abstr. 1972, 77, 549.

18. O Neal, J. B.; Rosen, H.; Russel, P. B.; Adams, A. C.; Blumenthal, A. J. Med. Pharm. Chem. 1962, 5, 617; Chem. Abstr. 1962, 57, 9168c.

19. Kurzer, F. Org. Compd. Sulphur, Selenium, Tellurium, 1974, 4, 417.

20. Thomas, J. Ger. Pat. 2,403, 357/1974, Chem. Abstr. 1974, $81,136153 \mathrm{~g}$.

21. Yale, H. L.; Losee, K. J. Med. Chem. 1966, 9, 478.

22. Turner, S. R. Colman Products Ltd. Ger. Pat. 1978, 2, 727, 146; Chem. Abstr. 1978, 88, 105357s.

23. Hodogaya, Chemical Co. Ltd., Jap. Pat. 1980, 80, 27024; Chem. Abstr. 1980, 93, 232719q.

24. Hakko Chem. Ind. Co. Ltd., Brit. Pat. 1,266,542/1972; Chem. Abstr. 1972, 77, 5474g.

25. Singh, H.; Yadav, L. D. S. Agric. Biol. Chem. 1976, 40, 759.

26. Misato, T.; Ko, K.; Honma, Y.; Konno, K.; Taniyama, E. Inst. Phys. Chem. Res. Jap. Pat. 1977, 772508; Chem. Abstr. 1977, 87, 147054.
27. Imramovsky; S. Polanc; J. Vinsova; M. Kocevar; J. Jampýlek; Z. Reckova; J. Kaustova; Bioorg. Med. Chem. 2007, 15, 2551.

28. H. Marrakchi; G. Laneelle; A. Quemard, Microbiology 2000, 146, 296.

29. (a) Joshi, R. S.; Mandhane, P. G.; Diwakar, S. D.; Gill, C. H., Ultrason. Sonochem. 2010, 17, 298 (b) Joshi, R. S., Mandhane, P. G., Badadhe, P. V., Gill, C. H.; Ultrason. Sonochem. 2011, 18, 735 (c) Mandhane, P. G.; Joshi, R. S.; Nagargoje, D. R.; Gill, C. H.; Tett. Letts. 2010, 51, 1490 (d) Joshi, R. S.; Mandhane, P. G.; Dabhade S. K.; Gill, C. H. J. Chin. Chem. Soc. 2010, 57, 1227 (e) Joshi, R. S.; Mandhane, P. G.; Shaikh, M. U.; Kale, R. P.; Gill, C. H. Chin. Chem. Lett. 2010, 21, 432.

30. (a) Joshi, R. S.; Mandhane, P. G.; Diwakar, S. D.; Dabhade, S. K. Gill, C. H., Bioorg. Med. Chem. Letts. 2010 , 20, 3721 (b) Joshi, R. S., Mandhane, P. G., Khan, W., Gill, C. H. Bull. Korean Chem. Soc. 2010, 31, 2341. (c) Joshi, R. S., Mandhane, P. G., Dabhade, S. K., Gill, C. H.; Green Chem. Lett. Rev. 2010, 3(3), 191 (d) Joshi, R. S.; Mandhane, P. G.; Khan, W.; Gill, C. H. J. Het. Chem. DOI 10.1002/ jhet.653.

31. Christine, H. F.; Michael, H. C. Antimicrob. Agents Chemother. 1986, 29, 386 . 